\title{
Effects and Consequences of Media Technology on Learning and Innovative Educational Strategies
}

\author{
Giusi Antonia Toto \\ Università degli Studi di Foggia, Foggia, ITALY \\ (D) 0000-0001-5538-5858 sc 57200033184 \\ giusy.toto@unifg.it
}

\author{
ARTICLE INFO \\ Received: 21 August 2018 \\ Accepted: 16 October 2018 \\ Published: 21 December 2018 \\ DOI: https://doi.org/10.29333/ojcmt/3988 \\ ABSTRACT
}

The use of media technology is a possibility of growth and at the same time an educational problem not to be underestimated because of the extreme pervasiveness and diffusion among adolescents. Many of the studies conducted so far, although not validated in the literature, suggest that Internet abuse would be related to dysfunctional and compulsive behaviours, tolerance and impoverishment of the functioning of the individual. This increases the risk of the onset of feelings of loneliness, depressive moods and low selfesteem. The purpose of this article is to examine some aspects of the positive and negative effects of technology in education and socialization of today's adolescents and the opportunity to use technology during text comprehension tasks (in this case on a geographical track) through a pilot study on Italian adolescents (age $\mathrm{m}=12.11$ ). Methodologically, in the first part of the document, a systematic review of the literature of the issues presented in the second part of the experience described, tools used and results obtained was carried out. The conclusions of this research are that the way to overcome the problem of the negative effects of technology is to exploit its educational potential, finalizing it to the construction of meanings and social relationships. In the study conducted, the students took into consideration the use of the highly motivating and stimulating technological tool even if, the general interpretation was better in the print texts than in the digital reading.

Keywords: media technologies, learning objects, innovative educational strategies, technological addiction, applied psychology

\section{INTRODUCTION}

In the process of building the identity of adolescents Digital Technologies play a fundamental role in terms of the attribution of meaning and social interaction (Rahmawati, et al., 2018). Today's teenagers called digital natives live in an environment whose boundaries are modified by the constant presence of social networks. In this environment the cognitive, emotional and relational dimensions undergo a redefinition based on the fusion between real and virtual (Toto, 2017a).

In the digital context, therefore, the development of adolescent identity must experience new possibilities for relationships and new needs for change; the need for new relationships oscillates between the need for interpersonal skills of a satisfactory selfimage (Fanchi, \& Scussolin, 2015). Ongoing research finds both positive effects in children and adolescents, among which an increase in motivation, creation of extended social 
networks, educational success, etc. both the impairment of mental health, emotional impetuosity, aggressive, intolerant and selfish behaviour towards parents or friends (Hyseni Duraku, 2018). According to the literature, in fact, it is possible to identify a dual attitude towards the technology that expresses the negative or positive judgment on its use: on the one hand, technology is considered an indispensable educational tool for the individual, it would improve the quality of learning; on the other hand, it could lead to the development of different forms of addictions and overlaps with other already known forms of behavioural addictions (Young, 1999).

Contrary to what is claimed by the pervasive use of media technology, the adolescent who 'plays' online is led to isolation and individualism, reduces social relationships but above all sports and physical activity, with obvious consequences for physical health in the particular growth phase. Many of these studies suggest that the precursors of internet abuse are loneliness, emptiness and the difficulty in perceiving reality (Kashiha, 2018; Wichstrøm, et al. 2018). For this reason, it is also necessary for education to strengthen the mechanisms to preserve one's health. The aim of this article in the first part was to find in the literature the effects of digital technologies on adolescence and possible proposals for resolution. In the second part of this article the opportunity to use media technology in the educational field during the text comprehension activities (in this case on a geographical task) was evaluated through a pilot study on Italian adolescents.

\section{METHODOLOGY OF RESEARCH}

A systematic review of the existing literature was adopted to search for articles in the main international databases (Google Scholar, PsycInfo and Scopus) on the issue of technological disadvantages among adolescents and on the relationships between cognitive abilities and prevention strategies, using, in English and in Italian, just the terms "Technology", "Learning objects", "Innovative Educational Strategies", Adolescent and "Technological addiction" as keywords. Following the Prisma guidelines (Moher, Liberati, Tetzlaff \& Altman, 2009) a systematic literature review process has been conducted: the literature search is followed by an evaluation of the titles and abstracts based on the research idea that although the evidence scientific relationships draw a strong relationship between technological dependence and adolescence, it is equally true that prevention and education to health and sport can reverse this trend, that is to make adolescents responsible users and not just passive users (identification).

Bibliographic research and evaluation for the inclusion of publications was conducted based on the research question expressed in the introduction. Regarding the inclusion and exclusion criteria, articles were selected in peer reviewed journals, books or book chapters in English or Italian that aimed to describe or evaluate the dimensions and variables expressed in the above-mentioned research idea (screening). All publications that dealt with addiction only in general, and those publications whose complete format (Relevance) could not be found were excluded. The time limit for the year of publication has been set for the last 5 years, so the articles have been selected since 2013. For the inclusion of the contributions, a qualitative summary of the most relevant information was also conducted with comparisons between the various publications without carrying out a quantitative analysis in the meta-analysis format. The process of inclusion of studies in the systematic review is described in Figure 1. After the elimination of duplicates, the research identified 612 studies consistent with the research idea. Subsequently on the basis of the title and the abstract 451 were excluded because they were not relevant. Of the 210 with full text 104 they met the inclusion criteria. 


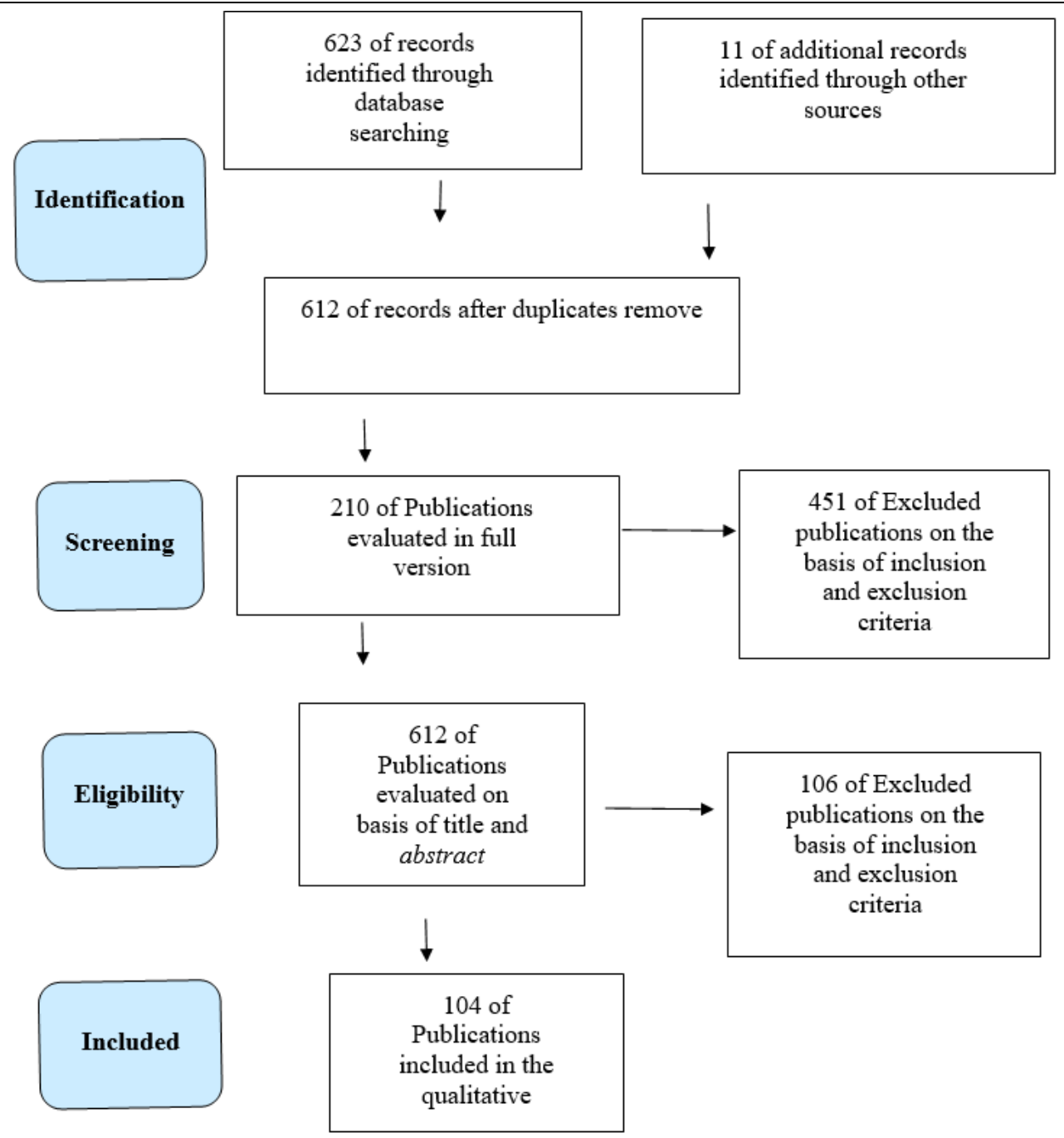

Figure 1. PRISMA Flow Chart of the selection process

\section{RESULTS AND RESEARCH}

This section presents the main contents of the literature on the topic. From this analysis emerge three main research strands:

1. Internet abuse produces effects comparable to those of substance addictions, both in terms of social behaviour and in terms of effects on the individual;

2. The negative effects of digital technologies are linked to the increase / worsening of feelings of loneliness, depression and low self-esteem;

3. The positive effects on education mainly concern the motivation and socialization of adolescents.

Regarding the first theme, that of dependence on technology (1), literature does not distinguish the effects of dependence on the basis of the subjects' age, but unites them by describing them as prey to a doubling condition, in which real experiences are they overlap with virtual ones, and in these they manifest their desires, research and the need for freedom. The studies define adolescents, who are particularly at risk for the development of this pathological disorder due to two characteristics inherent in adolescents themselves: they, for the development phase they live are looking for new experiences and sensations and are able to engage in adventurous and dangerous situations (Sensation seek). For this constitutive dimension they are the most vulnerable to the attractive characteristics of 
the technologies, which represent the risk factors of the beginning of the aforementioned dependencies. In addition to the search for new experiences in adolescence, impulsivity, considered as a personality trait, also plays a risk factor in the development of a pathological disorder (Logue, 1995; Shaw et al., 2006).

On the other hand, the review conducted revealed that emotional intelligence reduces vulnerability to inequality and thus becomes a protective factor against social anxiety, isolation and consequently addiction (Ambad, et al. 2017). The results reported in the selected literature have focused on different forms of technological dependence and the possible causes that can predict the onset of these dynamics in young adults and finally on the possible educational perspectives of resolution. Already the inclusion of Internet Gaming Disorder (IGD) in Section III of the Fifth Edition of the Diagnostic and Statistical Manual of Mental Disorders has increased the interest of researchers in the development of new standardized psychometric tools for the assessment of this disorder, but research on dependence of social networks, even though it has considerably increased over the last decade, has not yet validated tools nor a univocal definition in the international diagnostic literature. Starting from the previous discourse and analysing the second topic (2) traced according to Doumen et al. (2012). The general associations observed between attachment styles and identity factors would be related to online dependencies. In this direction, studies have shown the predictive role of the attachment style in the "Excessive use of social network sites (Aharony, 2016; Taga, et al. 2016). Specifically, affiliated individuals have large social networks and more social ties (Hart, et al. 2012), affiliated people use Facebook more frequently and are constantly worried about how they are perceived on Facebook (Lin, 2016), and the individuals characterized by the avoidance attachment style show little interest in Facebook (Oldmeadow et al., 2013). Monacis et al. (2017) has analysed for the first time the extent to which identity styles and attachment orientations can provoke the three types of online addiction (i.e., Internet Addiction, internet gaming addiction, and social media addiction). The results have shown that Internet, gaming and social media addictions have been predetermined by a common risk and protective factors: among identity styles "informational" and "diffusion-avoiding" styles are considered risk factors, while normative style is a protective factor. Among attachment dimensions, the "secure" attachment orientation is a protective factor, since it negatively predicts the three online addictions. These results highlight the important role played by identity formation in the development of technological addictions and confirm the different role plays by attachment styles. Previous studies have defined Internet addiction as a generic term that includes a wide range of online issues (Kuss, et al. 2017; Pontes \& Griffiths, 2017), for example, dependence on computer games or Internet gambling, online shopping, social network addiction, cellular dependency.

The third part of the current overview (3) analyses the value of media technology in educational context and how same educational strategies could promote a positive humancomputer interaction. The children of the present generation interact with a computer or more generally with technological tools before being able to swim, ride a bicycle or tie their shoes (Geist, 2014). New media, including social media, have increased the chances of reconsidering dependencies and tracing common coping styles by increasing the number of addicted subjects to be reached. Literature indicates (Toto, 2019) that the use of preventive measures is effective in treating addictions, in fact, a healthy lifestyle-based approach is strongly advocated, since it is aimed at affecting personality and personal choice components that have an impact on health and social responsibility. The various strategies have been very successful in promoting sports and physical education as a flyer 
of physical and mental wellbeing and precursor to a negative and stable attitude toward bad habits. Even though we are aware of the seriousness of the problem among people, and although the need for systematic preventive interventions is needed, educators show low self-esteem to carry out such interventions and help students (Karpov et al., 2015). The same problem is denounced by the family, committed to having educated subjects in a complex phase of the growth, in a neoliberal context that has produced a strong labour market crisis. The goal is to achieve a dialogue and collaboration between these two systems: the educational and parental system should share knowledge and communication through functional meetings (Limone, 2012).

As for the experiments using technological teaching methods, the literature describes how technology has been transformed from "information container to content vehicle and finally into interactive space" (Ligorio, 2010). The contemporary lines of research can not only adapt the new technologies to the classic contents of the disciplines, but question, experiment and provide transversal models that can be used in different educational contexts. Media Education, with particular reference to the relationship between technologies, digital media and learning process, is concentrating the contents of its research on the retroactive effects that technology has produced on the methods, contents and intelligence of those who use it daily (Filippello et al., 2014). Multimedia classes in school didactic practice beyond the use of technological tools, require software and innovative teaching strategies. The spread of this practice can be found in laboratories, and in the most disparate disciplines from literature to art, from technology to philosophy. However, there are many hesitations to introduce media technology in the classroom (Toto, 2017b). Many of these hesitations can be attributed to the lack of knowledge of the programs or the existence of teacher training courses. Technology has created an absurdity, even students without traditional knowledge and skills can easily express their ideas and participate in class activities even if they are culturally disadvantaged. However, in the analysed literature the media technology has not always been followed by the students slavishly: according to Legrottaglie and Ligorio (2014), when students are asked to express their preference between teacher and computer feedback, they have replied that they prefer a human teacher to feedback from the computer program. This preference shows that the relational and emotional aspect of education has not yet been achieved by technological tools.

Assuming that technology has an effect on enthusiasm, motivation, performance and information retrieval, a recent study has sought to combine music, technology and scientific studies in an interdisciplinary perspective (Yoon \& Kim, 2017). This experiment, conducted on a group of teachers and students, showed that while students were developing their songs, they had experimented with a process to practice science, including scientific concepts, and positively improved attitudes towards science. The success of this study on an adult audience demonstrates the added value of interdisciplinary and educational technology in learning processes, if adequately supported by appropriate technologies and its interdisciplinary approach.

\section{PILOT STUDY}

Participants in this study were 17 students. The sample was $64.7 \%$ of women with a mean age of 12.11 years. Our decision to focus on middle school students was based on various considerations related to the existing literature, the nature of the task and the texts read. Firstly, a recent review of the literature related to digital and printed reading indicated that over $75 \%$ of the identified studies concerned readers of lower secondary 
school (Singer, 2014; Singer \& Alexander, 2017a), therefore, in order to confirm the results of the current survey. On the existing literature, we believed that digital natives would better serve our goals. Moreover, individuals of this age group adapt to the digital natives profile (Prensky, 2001). As a result, we expected this population to be particularly convinced of digital reading, compared to the press, media. Furthermore, we have chosen physical education texts, which present images, texts and graphs that we consider relevant for middle school students. In this study four physical education texts on the alimentation in adolescent were used. This theme area was chosen for this reason: the topic of physical education was relevant to the participants' lectures without being treated beforehand.

Table 1. Participants

\begin{tabular}{cc}
\hline Male & Female \\
\hline 6 & 11 \\
$35.30 \%$ & $64.70 \%$ \\
\hline
\end{tabular}

Prior to administration, the four steps were modified to ensure that the frequencies of key points and other relevant information were kept constant between the texts. Consequently, each passage is centred around a clear main idea, furthermore each passage contained four key points, which were units of ideas based on the text or phrases directly linked to the main idea. For the study, each participant read four texts in total: two texts for each medium (i.e., digital and print). We were interested in examining the effects of the medium (i.e., digital and printed) on students' understanding. Before reading, participants were told that they would read a text and ask questions about the main idea and the key points. To assess their understanding, the students constructed concept maps with the main idea at the centre and the key points around. The scoring rules were determined a priori for the reading comprehension measure. The main idea was given a score of 1 point and the key points a score of 2 . The first and second text, as well as the third and fourth text, had the same total scores. The first text can score the maximum score of 8 per participant, the third text maximum score 10 . The internal reliability index of this instrument was excellent, ie equal to $\alpha=.85$.

Table 2. Results emerged

\begin{tabular}{|c|c|c|c|c|}
\hline & Digital & digital & print & print \\
\hline & Male & female & male & female \\
\hline First text & - & - & 45.1 & 86.2 \\
\hline Second text & 44.8 & 85.9 & - & - \\
\hline Third text & - & - & 76.2 & 109 \\
\hline Fourth text & 76.1 & 108.7 & - & - \\
\hline
\end{tabular}

Student's t test was used for independent samples in order to analyse the differences between males and females in each score on the scale. There were no significant differences ( $p>.05)$ between the two groups and therefore the gender had no effect on the variables under study. As a means of assessing students' calibration, we asked them to judge their performance under digital and print mediums. Specifically, the students answered the following question: In which medium do you believe you performed best? a. Digital b. Print c. No difference.

Table 3. Preferences for medium

\begin{tabular}{lcc}
\hline & male & female \\
\hline Digital & $40.40 \%$ & $35.40 \%$ \\
Print & $43.00 \%$ & $56.50 \%$ \\
No Difference & $16.60 \%$ & $9.10 \%$ \\
\hline
\end{tabular}

Gender effects were examined through t-test analyses. The results show that female have achieved a higher score in the preferences of the print medium in their comprehension tasks. 


\section{CONCLUSIONS}

Technology has structurally changed the relationships and the socio-cultural context in which individuals move; the diffusion of media technology has also produced contrasting effects in vast areas of human existence, including the world of education. In this study the main positive and negative effects studied in the specialized literature have been traced. It emerged that the studies focus the attention on three main aspects: 1 ) Internet abuse produces dysfunctional, compulsive behaviours, tolerance and impoverishment of the individual's functioning; 2) the digital technologies increase feelings of loneliness, depression and low self-esteem; 3) the positive effects of technology in education and on the socialization of adolescents. Specifically, the dependence on the technology is more studied for the effects on the subjects, studies on the causes are still experimental, even reported in their updates in this study. The second aspect investigated concerns the negative effects of technologies on cognitive and emotional dimensions; many of these studies suggest that internet abuse would be related to loneliness, vacuum, feelings of depression, difficulty in perceiving reality (Lee, et al. 2014). The daily use of the Internet is linked to people's social contexts, that is, the result of the lack of social support from their family members can facilitate the onset of technological addictions, since social contacts and reinforcement gained on the Internet can lead to an increase in the desire to maintain a 'virtual' social life. As a result, when adolescents develop social media addiction, they have little time and energy to keep active social contacts in their daily lives (Ponticorvo, \& Miglino, 2018). This phenomenon increases the risk of the onset of feelings of loneliness, depressive moods and low self-esteem (Lin et al., 2017). Finally, the use of media has already had significant consequences on teaching methods and on the practice of building and transmitting knowledge, but this should not ignore the necessary knowledge of the students' cultural background, which translates into terms of communication, relationship and construction of the identities and dynamics of social behaviours mediated by digital technologies (Vayola, 2016).

The second part of this study was motivated by the diffusion of digital media in the early school life of young people. Literature shows that digital natives prefer to read on digital media, perhaps because of the ease and accessibility that the Internet offers. Due to the ever-increasing presence of technologies inside and outside the classroom (Stephens, 2014), there has been an increase in research in the area of reading in digital media (Singer \& Alexander, 2017a; Wondemtegegn, 2018). However, research that specifically examines reading comprehension has produced mixed results in terms of which means are best for understanding text. While some studies have found that steps can be read more quickly in digital media (Kerr \& Symons, 2006), other studies have found greater advantages in reading comprehension (Mangen et al., 2013). In the present study, according to what has been shown in the literature (Singer \& Alexander, 2017b) in the comprehension of the text, the use of printed support is weaker than digital support. However, when asked digital natives if they prefer to read text in digital or printed format, they prefer printed texts (52.9\%). Regarding the first analysis, I found a different answer in terms of gender that is between men and women. Specifically, it emerged that men prefer printing effort in text tasks, as far as men are concerned

The fluidity of communication processes modified by educational technologies clashes with physical environments that are not able to respond to this continuous evolution and requires flexible solutions, able to satisfy contexts and educational activities that are always different to facilitate the student's active exploration (Junko, 2015; Limone, 2012). The existence of a positive correlation between learning and positive emotional 
involvement demonstrates the role of cognitive, affective and relational components in online teaching aimed at facilitating learning and enriching the educational relationship that can also be established on a virtual level. Positive emotional involvement is the aspect that most needs to be increased in educational technologies, in fact, learning improves when one is also emotionally involved in virtual interpersonal perception (Makransky \& Lilleholt 2018; Yakar, 2017). A possible solution to the problem may derive from prevention campaigns or educational activities that dissolve from solitude and isolation moments. Therefore, health education is important, in a broader sense: a healthy lifestyle-based approach is strongly advocated. The promotion of an interactive use of technology for example in physical sports and physical education could represent a flywheel of physical and mental wellbeing and precursor to a negative and stable attitude toward bad habits, as well as all digital creativity-focused activities implying a mental and social openness of adolescent.

\section{REFERENCES}

Aharony, N. (2016). Relationships among attachment theory, social capital perspective, personality characteristics, and Facebook self-disclosure. Aslib Journal of Information Management, 68(3), 362-386. https://doi.org/10.1108/AJIM-01-20160001

Ambad, S. N. A., Kalimin, K. M., \& Yusof, K. M. A. A. K. (2017). The Effect of Internet Addiction on Students' emotional And Academic Performance. e-Academia Journal, 6(1).

Doumen, S., Smits, I., Luyckx, K., Duriez, B., Vanhalst, J., Verschueren, K., \& Goossens, L. (2012). Identity and perceived peer relationship quality in emerging adulthood: The mediating role of attachment-related emotions. Journal of Adolescence, 35(6), 1417-1425. https://doi.org/10.1016/j.adolescence.2012.01.003

Fanchi, M., \& Scussolin, A., (2015). Strange games. Rete, esperienze ludiche e processi di rigenerazione dello spazio urbano (p.139-152). In: Catolfi A., \& Giordano F. (eds.). L’immagine videoludica. Cinema e media digitali verso la gamification.

Filippello, P., Sorrenti, L, Cuzzocrea, F., Nuzzaci, A., \& Larcan, R. (2014). The Subtle Sound of Learning: What Are the Roles of the Self-esteem, Decision-Making, and Social Skills in Adolescents' Academic Performance?. Us-China Education Review, 4, $73-85$.

Geist, E. (2014). Using tablet computers. Young Children, 69(1), 58-63.

Hart, J., Nailling, E., Bizer, G. Y., \& Collins, C. K. (2015). Attachment theory as a framework for explaining engagement with Facebook. Personality and Individual Differences, 77, 33-40. https://doi.org/10.1016/j.paid.2014.12.016

Ho, W. C. (2004). Use of information technology and music learning in the search for quality education. British Journal of Educational Technology, 35(1), 57-67. https://doi.org/10.1111/j.1467-8535.2004.00368.x

Hyseni Duraku, Z., Kelmendi, K., \& Jemini-Gashi, L. (2018). Associations of psychological distress, sleep, and self-esteem among Kosovar adolescents. International Journal of Adolescence and Youth, 1-9. https://doi.org/10.1080/02673843.2018.1450272

Junco, R. (2015). Student class standing, Facebook use, and academic performance. Journal of Applied Developmental Psychology,36, 18-29. https://doi.org/10.1016/j.appdev.2014.11.001 
Online Journal of Communication and Media Technologies, 2019

Karpov, V. Y., Eremin, M. V., Petrova, M. A., Alifirov, A. I., \& Skorosov, K. K. (2015). Modern approaches to preventing drug addiction by means of physical activity and sports. Biology and Medicine, 7(5), 1-4.

Kashiha, H. (2018). Malaysian ESL Students' Perception of Metadiscoure in Essay Writing. Online Journal of Communication and Media Technologies, 8(3), 193 201. https://doi.org/10.12973/ojcmt/2650

Kerr, M. A., \& Symons, S. E. (2006). Computerized presentation of text: Effects on children's reading of informational material. Reading and Writing, 19(1), 1-19. https://doi.org/10.1007/s11145-003-8128-y

Kuss, D. J., Griffiths, M. D., \& Pontes, H. M. (2017). Chaos and confusion in DSM-5 diagnosis of Internet Gaming Disorder: Issues, concerns, and recommendations for clarity in the field. Journal of Behavioral Addictions,6(2), 103-109. https://doi.org/10.1556/2006.5.2016.062

Lee, Y. K., Chang, C. T., Lin, Y., \& Cheng, Z. H. (2014). The dark side of smartphone usage: Psychological traits, compulsive behavior and technostress. Computers in Human Behavior, 31, 373-383. https://doi.org/10.1016/j.chb.2013.10.047

Legrottaglie, S., \& Ligorio, M. B. (2014). The use of technology at school: teachers' point of view. Italian Journal of Educational Technology, 22(3), 183-190.

Ligorio, M. B. (2010). Tecnologia e apprendimento. Paradigmi. Rivista di critica filosofica, 1, 181-187. https://doi.org/10.3280/para2010-001015

Limone, P. (2012). Le direzioni della scrittura scientifica: digitale, collaborativa, distribuita. Pedagogia Oggi, 89-106.

Limone, P. (2012). Ambienti di apprendimento e progettazione didattica. Proposte per un sistema educativo transmediale. Roma: Carocci.

Lin, J. H. (2016). Need for relatedness: A self-determination approach to examining attachment styles, Facebook use, and psychological well-being. Asian Journal of Communication, 26(2), 153-173. https://doi.org/10.1080/01292986.2015.1126749

Logue, A. W. (1995). Self-control: Waiting for Tomorrow for What you Want Today. (Prentice Hall: Englawood Cliffs).

Makransky, G., \& Lilleholt, L. (2018). A structural equation modeling investigation of the emotional value of immersive virtual reality in education. Educational Technology Research and Development, 1-24. https://doi.org/10.1007/s11423-018-9581-2

Mangen, A., Walgermo, B. R., \& Brønnick, K. (2013). Reading linear texts on paper versus computer screen: Effects on reading comprehension. International Journal of Educational Research, 58, 61-68. https://doi.org/10.1016/j.ijer.2012.12.002

Moher, D., Liberati, A., Tetzlaff, J., Altman, D. G., \& Prisma Group. (2009). Preferred reporting items for systematic reviews and meta-analyses: the PRISMA statement. PLoS medicine, 6(7), e1000097. https://doi.org/10.1371/journal.pmed. 1000097

Monacis, L., De Palo, V., Griffiths, M. D., \& Sinatra, M. (2017). Exploring individual differences in online addictions: The role of identity and attachment. International Journal of Mental Health and Addiction, 15(4), 853-868. https://doi.org/10.1007/s11469-017-9768-5

Oldmeadow, J. A., Quinn, S., \& Kowert, R. (2013). Attachment style, social skills, and Facebook use amongst adults. Computers in Human Behavior, 29(3), 1142-1149. https://doi.org/10.1016/j.chb.2012.10.006 
Pontes, H. M., \& Griffiths, M. D., (2017). The development and psychometric evaluation of the Internet Disorder Scale (IDS-15), Addictive Behaviors, 64, 261268. https://doi.org/10.1016/j.addbeh.2015.09.0030306-4603

Ponticorvo, M., \& Miglino, O. (2018). Hyper activity books for children: how technology can open books to multisensory learning, narration and assessment. Qwerty, 13, 4661.

Prensky, M. (2001). Digital natives, digital immigrants. Part 1. On the Horizon, 9(5), 1-6. https://doi.org/10.1108/10748120110424843

Rahmawati, A., Soesilowati, E., \& Sanjoto, T. B. (2018). Adolescent Lifestyle of Gadget Users in Kudus City. Journal of Educational Social Studies, 7(1), 51-58.

Shaw, P., Greenstein, D., Lerch, J., Clasen, L., Lenroot, R., Gogtay, N., Evans, A., Rapoport, J., \& Giedd, J., 2006, Intellectual ability and cortical development in children and adolescents. Nature, 440, 676-679. https://doi.org/10.1038/nature04513

Singer, L. M. (2014). Reading across mediums. Presentation at a meeting at the Educational Psychology Colloquium, Department of Human Development and Quantitative Methodology, College Park, MD.

Singer, L. M., \& Alexander, P. A. (2017a). Reading across mediums: Effects of reading digital and print texts on comprehension and calibration. The journal of experimental education, 85(1), 155-172. https://doi.org/10.1080/00220973.2016.1143794

Singer, L. M., \& Alexander, P. A. (2017b). Reading on paper and digitally: What the past decades of empirical research reveal. Review of Educational Research, 87(6), 10071041. https://doi.org/10.3102/0034654317722961

Stephens, M. (2014). Beyond news: The future of journalism. New York: Columbia University Press.

Toto, G. (2018). From Educational Contexts to Addictions: the Role of Technology in Teaching Methodologies and in Prevention as an Educational Function. Journal of eLearning and Knowledge Society, 14(2), 203-202.

Toto, G. A. (2017a). The influences of musical learning on psycho-physical development, intelligence and technology. The online journal of educational technology, 16(1), 604610.

Toto, G. A. (2017b). The Role of The musical Learning in the Development of the socio and cognitive abilities. A review. The online journal of educational technology, 16(1), 801807.

Toto, G. A., \& Strazzeri I. (2019). Sport and physical education as prevention against technological addictions. Journal of Human Sport \& Exercise, 14(1) https://doi.org/10.14198/jhse.2019.141.11

Vayola, P. (2016). Risks and opportunities of digital technologies in the school. Reflections about planning the teacher's training.Form@ re, 16(2), 180.

Wichstrøm, L., Stenseng, F., Belsky, J., von Soest, T., \& Hygen, B. W. (2018). Symptoms of Internet Gaming Disorder in Youth: Predictors and Comorbidity. Journal of abnormal child psychology, 1-13. https://doi.org/10.1007/s10802-018-0422-x

Wondemtegegn, S. A. (2018). University Students' Perception and Utilization of Technology for Learning: The Case of Haramaya University. Online Journal of Communication and Media Technologies, 8(1), 130-149.

Yakar, G. (2017). An Alternative Method and Display for Content Analysis: Textual Visualization. Online Journal of Communication and Media Technologies, 7(December 2017 - Special Issue), 14-21. 
Yoon, J., \& Kim, K. J. (2017). Science Song Project: Integration of Science, Technology and Music to Learn Science and Process Skills. K-12 STEM Education, 3(3), 235-250.

Young, K. (1999). Internet addiction: Evaluation and treatment. Student British Medical Journal, 7, 351-352.

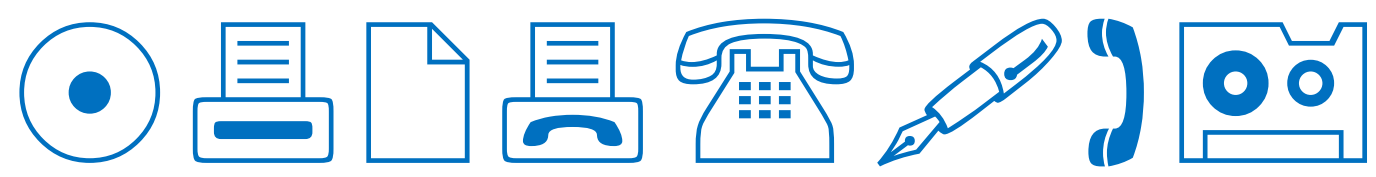

